\title{
Violência contra idosos e qualidade de vida relacionada à saúde: estudo populacional no município de São Paulo, Brasil
}

\author{
Violence perpetrated against the elderly and health-related quality \\ of life: a populational study in the city of São Paulo, Brazil
}

Daniel Rodrigues Machado (https://orcid.org/0000-0003-1255-7693) ${ }^{1}$

Miako Kimura (https://orcid.org/0000-0002-6062-112X)

Yeda Aparecida de Oliveira Duarte (https://orcid.org/0000-0003-3933-2179) ${ }^{2}$

Maria Lúcia Lebrão (https://orcid.org/0000-0002-2650-8684) ${ }^{2}$
${ }^{1}$ Escola de Enfermagem, Universidade de São Paulo (USP). Av. Dr Enéas Carvalho de Aguiar Cerqueira César. 05403 000 São Paulo SP Brasil. dani-machado@hotmail.com ${ }^{2}$ Faculdade de Saúde Pública, USP. São Paulo SP Brasil.

\begin{abstract}
The scope of this work was to identify the prevalence of domestic violence against non-institutionalized elderly individuals, and to establish if violence is an independent factor associated with the Physical Component (PC) and Mental Component (MC) scores of the Health-Related Quality of Life (HRQOL) of these elderly individuals. It is a cross-sectional epidemiologic and population-based investigation that is part of the SABE (Wellbeing, Health and Aging) study. A sample of 1,126 elderly individuals filled out the Short-Form 12 (SF-12) Health Survey, namely a generic instrument that assesses HRQOL through the Physical and Mental components. The prevalence of domestic violence against of the elderly was 10\% (CI 95\% 9.1-13.6). In the multiple anal$y$ ses, violence against the elderly was significantly associated to the MC $(\beta=-3.03 ; p=0.000)$ and to the PC $(\beta=-1.69 ; p=0.017)$ of HRQOL, independently of the sociodemographic, health, family support, and functional incapacity covariables. The prevalence of domestic violence was high and compromised the physical and mental health of the elderly.
\end{abstract}

Key words Quality of life, Elderly individuals, Mistreatment of the elderly
Resumo Objetivou-se identificar a prevalência da violência doméstica contra idosos não institucionalizados e verificar se esta é um fator independente associado aos Componentes Físico (CF) e Mental (CM) da Qualidade de Vida Relacionada à Saúde (QVRS) desses idosos. É um estudo epidemiológico transversal e de base populacional integrante do Estudo SABE (Saúde, Bem-Estar e Envelhecimento). A amostra foi de 1.126 idosos que responderam ao Short-Form 12 Health Related Survey (SF-12), instrumento genérico que avalia a QVRS em seus CF e CM. A prevalência da violência doméstica contra idosos foi de 10\% (IC 95\% 9,1-13,6). Na análise múltipla, a violência contra idosos permaneceu significativamente associada ao $C M(\beta=-3,03 ; p=0,000)$ e ao $C F(\beta$ $=-1,69 ; p=0,017)$ da QVRS, independente de covariáveis sociodemográficas, de saúde, de apoio familiar e de incapacidade funcional. A prevalência da violência doméstica foi elevada e comprometeu a saúde física e mental dos idosos.

Palavras-chave Qualidade de vida, Idoso, Maustratos ao idoso 


\section{Introdução}

Com o aumento expressivo da população idosa, a violência contra indivíduos mais velhos vem assumindo grandes proporções na sociedade moderna $^{1}$. Representa um problema de saúde pública de grande magnitude ${ }^{2,3}$ e constitui um dos tópicos mais relevantes das últimas décadas ${ }^{4}$, pois gera grande pressão sobre os sistemas de saúde, de segurança pública e de serviços sociais, bem como pode impactar diretamente na qualidade de vida das vítimas ${ }^{5}$.

A violência cometida contra idosos foi descrita pela primeira vez, em 1975, em publicação britânica como espancamento de avós. Desde então, tem sido tema de investigações científicas e alvo de ações governamentais em todo o mundo e, especificamente, no Brasil, desde a década de $1990^{2}$.

O primeiro estudo internacional de revisão sistemática sobre a prevalência da violência contra idosos detectou coeficientes de abuso entre 1,6\%, nos Estados Unidos da América, e 18\%, na Suécia e Dinamarca ${ }^{6}$. Uma metanálise recente com estudos de 28 países constatou que a prevalência da violência contra idosos foi de 15,7\%. No entanto, a falta de consenso na definição e medição da violência resultou em grandes variações nas taxas de prevalência dos 52 estudos incluídos na metanálise ${ }^{7}$. No Brasil, não se conhece a prevalência da violência contra idosos, pois existem poucas pesquisas de base populacional publicadas sobre $\mathrm{o}$ assunto.

A definição mais utilizada na literatura científica para o conceito "violência contra idosos" foi proposta pela Organização Mundial de Saúde $(\mathrm{OMS})^{8} \mathrm{e}$ adotada no presente estudo, na qual violência constitui uma ação única ou repetida ou ainda a ausência de uma ação devida, que cause sofrimento e angústia, e que ocorre em uma relação em que haja expectativa de confiança.

Ainda de acordo com a $\mathrm{OMS}^{2}$, algumas tipologias são utilizadas para designar as formas de violência mais praticadas contra a população idosa. A violência física refere-se à dominação induzida por drogas ou pelo uso da força física, resultando em dor ou lesão; a violência psicológica corresponde a infringir angústia mental; enquanto que a violência financeira ou material manifesta-se pela exploração imprópria ou ilegal do idoso ou no uso não consentido por ele de seus recursos patrimoniais e/ou financeiros.

Os idosos têm sido expostos a esses tipos de violência e eles ocorrem, predominantemente, de modo único ou combinado, no âmbito familiar².
Alguns fatores de risco em idosos vítimas da violência doméstica já foram descritos pela literatura científica, dentre os quais, merecem destaque: ter algum tipo de demência, deficiência física, depressão, solidão ou falta de apoio social; viver situações conflitivas com o cuidador; apresentar diminuição da capacidade funcional e cognitiva; bem como fazer uso de álcool ou drogas ilícitas9.

Independentemente da motivação, a ocorrência da violência poderá resultar em sofrimento desnecessário, lesão ou dor, perda ou violação dos direitos humanos, transtorno de estresse pós-traumático e somatização, tendendo a afetar severamente a saúde física e mental das vítimas ${ }^{10}$.

Alterações na saúde física e mental podem ser mensuradas pelas avaliações da Qualidade de Vida Relacionada à Saúde (QVRS), definida como o valor atribuído à duração da vida, modificado pelos prejuizos, estados funcionais, percepções e oportunidades sociais que são influenciados por doenças, dano, tratamento ou política de saúde $e^{11}$, não sendo, com frequência, diferenciada do estado funcional ou do estado de saúde (física e mental) de um indivíduo ${ }^{12}$.

As investigações que se propõem a identificar os fatores determinantes de boa ou má QVRS são cada vez mais necessárias e imprescindíveis, pois podem subsidiar a elaboração e a implementação de políticas públicas que visem à melhoria da saúde física e mental das pessoas. Nesse sentido, é válido mencionar que diversos pesquisadores nacionais e internacionais detectaram que indivíduos expostos à violência apresentaram piores escores de QVRS em relação aos não expostos ${ }^{13-15}$.

No entanto, essas pesquisas têm foco direcionado para a violência contra mulheres e indivíduos da população geral e, até o momento, não se tem conhecimento de nenhum estudo científico empírico que objetivou investigar a violência como fator independente associado à redução da QVRS de idosos.

No Brasil, investigações ajudaram a identificar variáveis socioeconômicas e de saúde associadas à QVRS de idosos da comunidade ${ }^{16,17}$. A detecção de outros fatores ligados à QVRS dessa população é primordial para o avanço do conhecimento científico.

Dessa forma, a presente investigação objetivou identificar a prevalência da violência doméstica contra idosos não institucionalizados do Município de São Paulo, assim como verificar se a violência é um fator independente associado aos escores dos componentes físico e mental da QVRS dos idosos. 


\section{Método}

Esta investigação é parte integrante do estudo SABE (Saúde, Bem-estar e Envelhecimento) e caracteriza-se como uma pesquisa epidemiológica transversal, com amostra de base populacional.

\section{O estudo SABE}

A Organização Pan-Americana da Saúde (OPAS) e a OMS coordenaram o estudo multicêntrico denominado SABE com o objetivo de detalhar as condições de vida e o estado de saúde de idosos residentes em sete centros urbanos da América Latina e Caribe. O estudo SABE teve início em 2000 e foi conduzido na Argentina, Barbados, Brasil, Chile, Cuba, México e Uruguai. No Brasil, uma amostra representativa de idosos não institucionalizados (60 anos ou mais) foi obtida no Município de São Paulo, tendo em vista o apoio institucional da Universidade de São Paulo (USP) e o financiamento da Fundação de Amparo à Pesquisa do Estado de São Paulo (FAPESP).

Somente nesse país, o SABE constitui-se em estudo longitudinal com quatro ondas (2000, 2006, 2010 e 2015), coordenado por uma equipe de pesquisadores do Departamento de Epidemiologia da Faculdade de Saúde Pública da USP. O detalhamento da técnica de amostragem do estudo SABE encontra-se descrito em publicações específicas ${ }^{18-20}$. Ressalta-se que, em 2010, a população de idosos residentes no Município de São Paulo era de 1.338.138 indivíduos ${ }^{21}$.

\section{Amostra e critério de exclusão}

Foi realizada uma análise transversal com os idosos brasileiros que participaram do estudo SABE em 2010. Dos 1.345 participantes, foram excluídos 219, cujos questionários foram respondidos por um informante auxiliar ou substituto. A adoção desse critério de exclusão é justificada pela premissa de que a qualidade de vida, principal desfecho do presente estudo, é um construto de natureza subjetiva e, portanto, deve ser aferida com base nas respostas do próprio indivíduo que está sendo avaliado ${ }^{22}$. Ademais, o informante auxiliar ou substituto poderia ser o provável agressor do idoso e, nesse contexto, a informação sobre a ocorrência da violência poderia ser omitida ou não fidedigna ${ }^{12,23}$.

Portanto, a amostra final do presente estudo constituiu-se de 1.126 idosos ( $\geq 60$ anos) não institucionalizados do Município de São Paulo.

\section{Coleta de dados}

Os dados foram coletados por meio de entrevistas individualizadas, face a face, realizadas nos domicílios dos idosos (em um local da residência que oferecesse privacidade) por uma equipe de entrevistadores, especialmente treinada para atuar no estudo SABE.

O questionário utilizado no estudo SABE está composto por doze seções que abrangem vários aspectos da vida do idoso, tais como: dados pessoais, avaliação cognitiva, estado de saúde, estado funcional, medicamentos, uso e acesso aos serviços, rede de apoio familiar e social, história laboral e fontes de receita, características da moradia, violência, antropometria, flexibilidade e mobilidade (disponível em http://www.fsp.usp.br/sabe/ quetionario.php).

\section{Variáveis dependentes}

Os escores do Componente Físico (CF) e Mental (CM) do SF-12 foram as variáveis dependentes deste estudo. Validado no Brasil em amostras clínicas $^{24}$ e na população geral2 ${ }^{25}$ o SF-12 está composto por 12 itens derivados do SF-36 (36-item Short Form Health Survey) ${ }^{26}$, um instrumento genérico de avaliação de QVRS que se encontra traduzido e validado em português ${ }^{27}$. As oito dimensões do SF-12 são: estado geral de saúde, capacidade funcional, aspectos físicos, dor, vitalidade, saúde mental, aspectos emocionais e aspectos sociais, sendo que as quatro primeiras formam o $\mathrm{CF}$ e as demais, o CM.

Os escores dos componentes do SF- 12 podem variar de 0 a 100\%, sendo as pontuações mais elevadas tradução de melhor percepção de saúde física ou mental e as mais baixas, o oposto ${ }^{24,25}$. No presente estudo, o coeficiente alfa de Cronbach foi de 0,77 para o CF e de 0,74 para o CM, valores indicativos de consistência interna satisfatória.

\section{Variáveis independentes}

Variáveis sociodemográficas e de saúde foram investigadas, incluindo sexo (masculino e feminino), idade (número de anos completos no momento da entrevista), raça/cor (branca, parda, preta e outras), coabitação (sozinho e acompanhado), escolaridade (anos de estudo no momento da entrevista) e suficiência de renda percebida (suficiente e insuficiente), sendo esta última variável acessada por meio da pergunta: $o$ (a) senhor(a) considera que tem dinheiro suficiente para cobrir suas despesas diárias? 
A percepção do estado geral de saúde (boa, regular, ruim) foi obtida por meio da indagação: o(a) Senhor(a) diria que sua saúde é muito boa, boa, regular, ruim ou muito ruim?. A categoria "boa" foi composta pelas respostas boa e muito boa, a categoria "regular" correspondeu à própria alternativa regular e a categoria "ruim", foi formada pelo agrupamento das respostas ruim e muito ruim.

Os dados sobre a variável dor (sim, não) foram acessados por meio de duas perguntas: 1) $o$ (a) Senhor(a) sente dor ou desconforto quando faz algum esforço físico ou movimento como, por exemplo, levantar e andar? e 2) o(a) Senhor(a) tem alguma dor há mais de 3 meses, que dói continuamente ou que vai e vem, pelo menos, uma vez por mês? Uma resposta afirmativa para qualquer uma dessas duas perguntas caracterizou o idoso como portador de dor.

O número de doença(s) crônica(s) (nenhuma, uma, duas ou mais) considerou as seguintes doenças: hipertensão (pressão sanguínea alta), doença cardíaca (ataque do coração, doença coronária, angina, doença congestiva ou outro problema cardíaco), acidente vascular encefálico (embolia, derrame, ataque, isquemia ou trombose cerebral), diabetes mellitus (níveis altos de açúcar no sangue), doença pulmonar crônica (como asma, bronquite ou enfisema), doença osteoarticular (artrite, reumatismo, artrose), neoplasia (excluindo os tumores menores de pele) e problema neurológico ou psiquiátrico. Os dados foram acessados por meio da pergunta: alguma vez um médico ou enfermeiro the disse que o(a) Senhor(a) tem hipertensão, quer dizer pressão sanguinea alta?, mudando o nome da doença ao final da frase.

O declínio cognitivo foi avaliado pelo MEEM (Miniexame do Estado Mental) ${ }^{28}$, modificado e validado no Chile $^{29}$ e traduzido para o português ${ }^{30}$. A pontuação do MEEM varia de zero a 19 e o declínio cognitivo é indicado por 12 ou menos pontos obtidos na versão modificada da escala ${ }^{29}$.

Para o estudo dos sintomas depressivos foi utilizada a Escala de Depressão Geriátrica Abreviada (GDS - 15 itens), validada no Brasil ${ }^{31}$. A pontuação do instrumento varia de 0 a 15 e 6 ou mais pontos alcançados na escala indicam que o idoso tem sintomas sugestivos de depressão ${ }^{31}$. O coeficiente alfa de Cronbach para a GDS foi de 0,69 , valor indicativo de consistência interna satisfatória.

A funcionalidade familiar foi avaliada por meio do instrumento denominado APGAR de
Família (Family Apgar), que no Brasil foi traduzido e adaptado para ser aplicado em idosos ${ }^{32}$. As cinco questões do instrumento permitem pontuação variando de 0 a 20 , e quanto mais elevado for o escore, melhor será a funcionalidade familiar. Neste estudo, os escores de 0 a 12 foram categorizados como família disfuncional e de 13 a 20 , família funcional ${ }^{16}$. Os cinco itens da escala mostraram alta consistência interna (coeficiente alfa de Cronbach $=0,89$ ), indicando a confiabilidade da medida.

Em relação à incapacidade funcional, avaliou-se, separadamente, a dificuldade para executar atividades básicas - $\mathrm{ABVD}^{33} \mathrm{e}$ instrumentais da vida diária - $\mathrm{AIVD}^{34}$ (nenhuma dificuldade, dificuldade em uma ou duas atividades, dificuldade em três ou mais atividades). As ABVD investigadas foram: usar o vaso sanitário, alimentar-se, banhar-se, vestir-se, transferir-se e atravessar um quarto caminhando. As questões sobre AIVD incluíram: utilizar um meio de transporte, fazer compras, cuidar do próprio dinheiro, usar o telefone e tomar medicamentos.

Neste estudo, a ocorrência da violência foi detectada por meio de um questionário utilizado em pesquisas científicas ${ }^{35-37}$ e recomendado pelo Ministério da Saúde do Brasil como subsídio técnico aos profissionais que atuam na atenção primária $^{38}$. Por meio desse questionário é possível identificar violência física, psicológica ou financeira contra idosos.

As perguntas do questionário de identificação de violência são as seguintes: 1) no último ano, alguma das pessoas que o rodeiam tem gritado com o Senhor sem razão?; 2) no último ano, alguma das pessoas que o rodeiam tem chamado por algum nome ou apelido que o Senhor não goste?; 3) no último ano, alguma das pessoas que o rodeiam tem usado ou mexido no seu dinheiro sem sua autorização?; 4) no último ano, alguma das pessoas que o rodeiam tem ameaçado em função de o Senhor não fazer o que querem que o Senhor faça?; 5) no último ano, alguma das pessoas que o rodeiam tem batido ou esbofeteado o Senhor?; 6) no último ano, alguma das pessoas que o rodeiam tem sacudido ou chacoalhado o Senhor?, e 7) no último ano, alguma das pessoas que o rodeiam tem roubado seu dinheiro ou algum pertence importante para o Senhor?

Para cada pergunta havia como possibilidade de resposta sim, não ou ignorado. Ressalta-se que uma resposta afirmativa (sim) para qualquer pergunta desse questionário bastava para caracterizar o entrevistado como vítima da violência. 


\section{Análise estatística}

Os dados foram analisados pelo software $\mathrm{R}$ versão 3.0.1, utilizando-se o pacote survey que permite incorporar aspectos referentes ao delineamento complexo de amostragem: estratificação não proporcional, sorteio de conglomerados e ponderação.

A estatística descritiva foi utilizada para a análise das variáveis independentes e dependentes da pesquisa. A confiabilidade dos instrumentos padronizados foi testada por meio da análise de consistência interna, utilizando-se o coeficiente alfa de Cronbach. O valor de referência adotado como aceitável (boa confiabilidade) foi igual ou maior a $0,60^{39}$. Calculou-se a prevalência da violência contra idosos com intervalo de $95 \%$ de confiança.

Foram ajustados modelos de regressão linear ponderados para explicar os escores dos CF e CM da QVRS. Foram selecionadas 14 variáveis independentes: sexo, idade, raça/cor, escolaridade, coabitação, suficiência de renda percebida, funcionalidade familiar, número de doenças crônicas, dor, sintomas depressivos, declínio cognitivo, dificuldade para executar ABVD e AIVD e exposição à violência. $\mathrm{O}$ método utilizado na análise de regressão foi o stepwise backward, adotando-se o critério de Akaike para a seleção do modelo final. A autocorrelação dos resíduos (colinearidade) foi testada pelo Teste de Durbin Watson, e a qualidade do ajuste do modelo foi representada pelo coeficiente de determinação $\left(\mathrm{R}^{2}\right)$. Todos os pressupostos desse tipo de regressão foram analisados. $\mathrm{O}$ nível de significância dos testes foi de 5\% e utilizou-se a correção de Rao-Scott para amostragem complexa.

\section{Aspectos éticos}

O estudo SABE foi aprovado pelo Comitê de Ética em Pesquisa da Faculdade de Saúde Pública da USP.

\section{Resultados}

Os idosos incluídos (Tabela 1) tinham média de idade equivalente a 69,2 anos $(\mathrm{dp}=7,4)$ e estudaram, em média, 5,7 anos $(\mathrm{dp}=4,4)$.

A prevalência de violência contra idosos foi de 10\% (IC 95\% 9,1 - 13,6). Os dados da Tabela 2 apresentam os escores dos componentes físico e mental da QVRS.

Observa-se na Tabela 2 que o escore do CM foi superior ao do CF da QVRS dos idosos. A
Tabela 1. Características sociodemográficas, de funcionalidade familiar, de estado de saúde e de incapacidade funcional dos idosos. São Paulo, Brasil, $2010(\mathrm{n}=1.126)$.

\begin{tabular}{|c|c|}
\hline Características & $\%$ \\
\hline \multicolumn{2}{|l|}{ Sexo } \\
\hline Masculino & 40,2 \\
\hline Feminino & 59,8 \\
\hline \multicolumn{2}{|l|}{ Raça/cor } \\
\hline Branca & 59,1 \\
\hline Parda & 28,9 \\
\hline Preta & 6,2 \\
\hline Outras & 5,8 \\
\hline \multicolumn{2}{|l|}{ Coabitação } \\
\hline Sozinho & 15,8 \\
\hline Acompanhado & 84,2 \\
\hline \multicolumn{2}{|l|}{ Suficiência de renda percebida } \\
\hline Suficiente & 57,3 \\
\hline Insuficiente & 42,7 \\
\hline \multicolumn{2}{|l|}{ Funcionalidade familiar } \\
\hline Funcional & 87,7 \\
\hline Disfuncional & 12,3 \\
\hline \multicolumn{2}{|l|}{ Percepção do estado de saúde } \\
\hline Boa & 51,5 \\
\hline Regular & 42,0 \\
\hline Ruim & 6,5 \\
\hline \multicolumn{2}{|l|}{ Número de doenças crônicas } \\
\hline Nenhuma & 14,7 \\
\hline Uma & 26,3 \\
\hline Duas ou mais & 59,0 \\
\hline \multicolumn{2}{|l|}{ Dor } \\
\hline $\operatorname{Sim}$ & 32,0 \\
\hline Não & 68,0 \\
\hline \multicolumn{2}{|l|}{ Sintomas depressivos } \\
\hline $\operatorname{Sim}$ & 17,7 \\
\hline Não & 82,3 \\
\hline \multicolumn{2}{|l|}{ Declínio cognitivo } \\
\hline Sim & 3,1 \\
\hline Não & 96,9 \\
\hline \multicolumn{2}{|l|}{ Dificuldade para executar ABVD } \\
\hline Nenhuma dificuldade & 75,8 \\
\hline Dificuldade em uma ou duas & 18,1 \\
\hline ABVD & 6,1 \\
\hline \multicolumn{2}{|l|}{ Dificuldade em Três ou mais } \\
\hline \multicolumn{2}{|l|}{ ABVD } \\
\hline \multicolumn{2}{|l|}{ Dificuldade para executar AIVD } \\
\hline Nenhuma dificuldade & 79,8 \\
\hline Dificuldade em uma ou duas AIVD & 15,9 \\
\hline Dificuldade em três ou mais AIVD & 4,3 \\
\hline
\end{tabular}

Tabela 3 mostra os modelos finais da análise de regressão múltipla para os escores do $\mathrm{CF}$ e $\mathrm{CM}$ do SF-12. 
Entre as 14 variáveis que compuseram o modelo inicial de regressão, sete foram preditoras independentes do CF da QVRS: dor, multimorbidade, necessidade de ajuda em ABVD e AIVD e violência. $O$ valor de $R^{2}$ ajustado indica que $45,8 \%$ da variabilidade do CF é explicada pelas variáveis que permaneceram no modelo final. Em relação ao $\mathrm{CM}$, continuaram como variáveis associadas: escolaridade, renda suficiente, funcionalidade familiar, sintomas depressivos, dor, multimorbidade, ajuda em AIVD e violên-

Tabela 2. Escores dos CF e CM da QVRS dos idosos. São Paulo, Brasil, 2010 ( $\mathrm{n}=1.126)$.

\begin{tabular}{lr}
\hline Componentes do SF-12 & Média $(\mathbf{d p})$ \\
\hline CF & $48,6(9,6)$ \\
CM & $55,2(8,2)$ \\
\hline
\end{tabular}

cia. Ressalta-se que a exposição à violência teve um coeficiente $\beta$ negativo de 3,03 e um nível de significância $\mathrm{p}=<0,001$, o que significa que ser exposto à violência reduz uma estimativa de 3,03 pontos o escore do CM do SF-12. O teste de Durbin Watson para os CF e CM do SF-12 indica que não houve colinearidade entre os resíduos.

\section{Discussão}

Embora a violência contra idosos seja um problema de saúde pública global ${ }^{3}$, há pouco conhecimento sobre esse tema em países menos desenvolvidos. Isso pode ser atribuído, entre outros motivos, à escassez de dados epidemiológicos sobre a violência contra idosos ${ }^{40}$. No Brasil, estudos documentaram prevalência da violência contra idosos variando de 10,1 a $21 \%$, sendo de $10,1 \%$ em Niterói ${ }^{41}$, 12,4\% em Florianópolis ${ }^{36}, 13,3 \%$ no

Tabela 3. Regressão linear múltipla para os escores dos CF e CM do SF-12. São Paulo, Brasil, 2010 ( $\mathrm{n}=1.126)$.

\begin{tabular}{|c|c|c|c|c|c|}
\hline Componentes & $\begin{array}{c}\text { Variáveis que permaneceram no } \\
\text { modelo final }\end{array}$ & $\begin{array}{c}\text { Coeficiente } \\
\boldsymbol{\beta} \\
\end{array}$ & p- valor & $\mathbf{R}^{2}$ & $\begin{array}{l}\text { Durbin } \\
\text { Watson }\end{array}$ \\
\hline \multirow[t]{13}{*}{$\mathrm{CF}$} & Constante & 55,58 & $<0,001$ & 0,458 & 0,054 \\
\hline & Raça/cor (Parda) & 0,44 & 0,364 & & \\
\hline & Raça/cor (Preta) & 0,48 & 0,588 & & \\
\hline & Raça/cor (Outras) & 0,59 & 0,519 & & \\
\hline & Renda suficiente & 0,80 & 0,070 & & \\
\hline & Dor & $-3,34$ & $<0,001$ & & \\
\hline & 1 doença crônica & $-0,76$ & 0,272 & & \\
\hline & 2 ou mais doenças crônicas & $-3,55$ & $<0,001$ & & \\
\hline & Ajuda em 1 ou 2 ABVD & $-4,44$ & $<0,001$ & & \\
\hline & Ajuda em 3 ou mais ABVD & $-13,48$ & $<0,001$ & & \\
\hline & Ajuda em 1 ou 2 AIVD & $-5,52$ & $<0,001$ & & \\
\hline & Ajuda em 3 ou mais AIVD & $-8,56$ & $<0,001$ & & \\
\hline & Violência & $-1,69$ & 0,017 & & \\
\hline \multirow[t]{14}{*}{$\mathrm{CM}$} & Constante & 52,26 & $<<0,001$ & 0,214 & 0,566 \\
\hline & Raça/cor (Parda) & 0,19 & 0,712 & & \\
\hline & Raça/cor (Preta) & 0,74 & 0,430 & & \\
\hline & Raça/cor (Outras) & $-0,38$ & 0,692 & & \\
\hline & Escolaridade & $-0,14$ & 0,007 & & \\
\hline & Renda suficiente & 1,29 & 0,006 & & \\
\hline & Funcionalidade familiar & 0,37 & $<0,001$ & & \\
\hline & Sintomas depressivos & $-3,97$ & $<0,001$ & & \\
\hline & Dor & $-1,03$ & 0,037 & & \\
\hline & 1 doença crônica & $-0,63$ & 0,381 & & \\
\hline & 2 ou mais doenças crônicas & $-1,53$ & 0,022 & & \\
\hline & Ajuda em 1 ou 2 AIVD & $-2,96$ & $<0,001$ & & \\
\hline & Ajuda em 3 ou mais AIVD & $-7,02$ & $<0,001$ & & \\
\hline & Violência & $-3,03$ & $<0,001$ & & \\
\hline
\end{tabular}


Rio de Janeiro ${ }^{40}, 14,4 \%$ em São Paulo ${ }^{40}, 17,8 \%$ em Porto Alegre ${ }^{42}, 20,8 \%$ no Recife ${ }^{35}, 20,9 \%$ em Uberaba $^{43}$ e $21 \%$ em Camaragibe ${ }^{44}$.

No presente estudo, $10 \%$ dos participantes referiram ter sofrido algum tipo de violência. Essa prevalência é maior do que a encontrada em estudos realizados na Europa ${ }^{45}(5,6 \%)$ e América do Norte $^{46}(8,1 \%)$ e também superior às estimativas de violência doméstica contra idosos divulgadas pela OMS $(4 \text { a } 6 \%)^{2}$, embora seja inferior às taxas encontradas na África e $(43,7, \%)^{47}$, Ásia $(49,1 \%)^{48}$ e em outras localidades no Brasil ${ }^{35,36,40-44}$.

Diferentes abordagens metodológicas, incluindo diferenças nos procedimentos de amostragem e nos instrumentos de coleta de dados utilizados, bem como os aspectos culturais envolvidos podem explicar essa variação da prevalência da violência contra idosos. Estudos adicionais são necessários para melhor investigar essa questão, de preferência utilizando um conjunto comum de procedimentos ${ }^{7,40}$.

É importante afirmar que a ocorrência da violência detectada no presente estudo provavelmente subestima a verdadeira prevalência desse agravo no Município de São Paulo, pois os métodos utilizados para a sua avaliação não envolveram informações de profissionais de saúde responsáveis pelos idosos, registros policiais, realização de exame físico, bem como pelo fato de que idosos vítimas de abusos graves provavelmente não aceitaram participar de um estudo da natureza do SABE.

A exclusão de 219 idosos cujos questionários foram respondidos por um informante auxiliar ou substituto também pode ter subestimado a prevalência da violência. Além disso, mencionase que alguns casos de violência podem não terem sido revelados pelos próprios idosos, tendo em vista que indivíduos mais velhos tendem a não relatar acontecimentos dessa natureza ${ }^{49}$.

Pesquisa conduzida em 204 municípios distribuídos nas cinco macrorregiões do Brasil (Sudeste, Nordeste, Sul, Norte e Centro-Oeste) registrou que $40 \%$ dos idosos vítimas de violência não relataram o fato a pessoas conhecidas ou a órgãos competentes ${ }^{49}$. A vergonha, o receio de retaliações do agressor, o medo do rompimento dos laços familiares, da perda da autonomia e do local onde reside, já que grande parte das vítimas vive com o agressor, são alguns dos fatores associados à omissão da ocorrência da violência ${ }^{10}$.

Dessa forma, a determinação fidedigna da prevalência da violência contra idosos é muito difícil ou até mesmo impossível de ser obtida, pelo caráter, muitas vezes, velado do problema ${ }^{2,10}$.
Ainda assim, os estudos que se propõem a identificar a ocorrência da violência contra idosos são de grande utilidade, pois ajudam a dar visibilidade ao problema e oferecem subsídios para a estimação da magnitude do fenômeno ${ }^{50}$.

A análise de regressão linear múltipla apontou que a violência contra idosos foi significativamente associada ao $\mathrm{CM}(=-3,03 ; \mathrm{p}=0,000)$ e ao CF ( $=-1,69 ; \mathrm{p}=0,017)$ da QVRS, independente de covariáveis sociodemográficas, de saúde, de apoio familiar e de incapacidade funcional. A violência contra idosos produz efeitos complexos e multidimensionais à saúde das vítimas, incluindo raiva, desmoralização, vergonha, humilhação, medo, isolamento social, insônia, falta de apetite, indigestão, solidão, tristeza e infelicidade diante da vida ${ }^{51}$.

Estudo transversal realizado com 2.021 ido$\operatorname{sos}(\geq 65$ anos) residentes em sete regiões da Irlanda encontrou, por meio de análise multivaria$\mathrm{da}$, que obter escore abaixo da média no CM da QVRS, aferida pelo SF-8, aumentou em mais de quatro vezes as chances de relato de qualquer tipo de violência ( $O R=4,51$, IC 95\% 2,22 - 9,14), ao passo que o CF da QVRS não foi significativamente associado ao aumento da chance de relatar violência ${ }^{52}$.

Todavia, é preciso ter cautela ao se comparar os resultados da presente pesquisa com os da irlandesa supracitada. Uma comparação entre as amostras desses estudos aponta diferenças em relação às características demográficas e culturais dos idosos. Cabe também destacar que a QVRS foi tratada com enfoques diferentes no plano de análise. Na presente pesquisa, a QVRS foi a variável desfecho, enquanto que no estudo irlandês os CF e CM da QVRS foram tratados como variáveis independentes potencialmente associadas ao desfecho violência contra idosos.

Portanto, nossos resultados evidenciam, pela primeira vez, que a violência é uma variável associada à redução do escore do CF e CM da QVRS de idosos, mesmo após o controle de fatores sociodemográficos, de apoio familiar, de saúde e de incapacidade funcional. Este resultado deve servir de alerta aos vários segmentos da sociedade, sobretudo aos profissionais de saúde e aos próprios indivíduos idosos. No caso dos profissionais da saúde, é preciso que muitos deles passem a reconhecer a violência como um problema também pertencente ao campo da saúde e abandonem, definitivamente, a posição de expectadores do fenômeno. Já os idosos necessitam ser alertados de que a omissão no relato de violência pode resultar em queixas e sintomas inespecíficos que dificul- 
tam o diagnóstico e o tratamento de distúrbios físicos e mentais resultantes da violência.

Embora o foco do presente estudo tenha incidido na relação entre a violência e os componentes físico e mental da QVRS de idosos, cabe ressaltar que outras variáveis também se mostraram significativamente associadas aos desfechos analisados. As associações com incapacidade funcional para atividades da vida diária, multimorbidade, dor e sintomas depressivos são congruentes com outros estudos ${ }^{16,53-56}$ e ratificam a necessidade de detecção e manejo de condições físicas e psíquicas que possam comprometer a QVRS de idosos.

Pesquisa de base populacional realizada no Município de São Paulo, utilizando o SF-12, identificou associação entre dificuldade para realizar ABVD e CF da QVRS de idosos. A chance para uma boa QVRS foi 87\% menor para os homens idosos com dificuldade para ABVD em relação àqueles sem dificuldades. Já as mulheres idosas com dificuldades para desempenhar ABVD tiveram suas chances para uma boa QVRS reduzidas em $73 \%{ }^{16}$.

No tocante à multimorbidade, pesquisa cuja amostra foi representativa da população idosa italiana não institucionalizada (33.744 indivíduos $\geq 60$ anos), detectou que a ocorrência de duas ou mais doenças crônicas afetou o CF e o CM dos idosos, mensurados pelo SF-12, sendo piores os escores do CF. No CF, os idosos com multimorbidade tiveram chance $73 \%$ menor para considerarem ter boa QVRS, comparados àqueles sem doença crônica. Já no CM, para os idosos com multimorbidade, a chance para uma boa QVRS foi $50 \%$ menor $^{54}$.

Diversas pesquisas identificaram a dor como variável associada à pior percepção da qualidade de vida entre idosos ${ }^{55-56}$. Pesquisa transversal e de base populacional realizada com 1.593 idosos residentes em Bagé, Rio Grande do Sul, documentou que os insatisfeitos com a vida tiveram uma prevalência duas vezes maior de sintomas depressivos quando comparados aos satisfeitos ${ }^{57}$.

O presente estudo apresenta algumas limitações. Em primeiro lugar, cita-se a opção pelo corte transversal, que não permitiu estabelecer causalidade entre exposição à violência e QVRS. Em segundo, embora a prevalência da violência seja alta, ela pode estar subestimada por razões já mencionadas. Em terceiro, não foi utilizado um instrumento de detecção da violência validado para uso no Brasil, e que fosse capaz de identificar outros tipos de violência não detectados nessa investigação (sexual e negligência) e de oferecer dados sobre a gravidade das agressões contra idosos. No entanto, a escolha do questionário sobre violência aplicado neste estudo tem utilização precedente em outras pesquisas ${ }^{35-37}$.

Por fim, destaca-se que a representatividade da amostra é uma fortaleza do estudo. A técnica de amostragem randomizada, baseada em setores censitários, garantiu uma amostra representativa dos idosos não institucionalizados residentes no maior município das Américas, em termos populacionais. Essa pesquisa preenche uma lacuna do conhecimento e estabelece bases para futuros estudos sobre a exposição à violência ao longo do tempo e seu impacto na saúde física e mental dos idosos.

\section{Conclusões}

Este estudo detectou prevalência de $10 \%$ da violência contra idosos. A análise de regressão múltipla evidenciou que a violência comprometeu a saúde física e mental dos idosos, independente das covariáveis sociodemográficas, de saúde, de apoio familiar e de incapacidade funcional analisadas neste estudo. É imprescindível que a violência contra pessoas mais velhas seja fenômeno merecedor de atenção dos profissionais de saúde, gestores e pesquisadores que atuam na área de atenção aos idosos.

\section{Colaboradores}

DR Machado realizou a proposta deste trabalho, a organização, análise e interpretação dos dados, a redação e aprovação da versão final do manuscrito. M Kimura participou da organização, análise e interpretação dos dados e aprovação da versão final do manuscrito. YAO Duarte e ML Lebrão participaram da revisão e da aprovação da versão final do manuscrito. 


\section{Referências}

1. Ho CS, Wong SY, Chiu MM, Ho RC. Global prevalence of elder abuse: a meta-analyses and meta-regression. East Asian Arch Psychiatry 2017; 27(2):43-55.

2. Organização Mundial de Saúde (OMS). Relatório mundial sobre violência e saúde. Genebra: OMS; 2002.

3. Reichenheim ME, Souza ER, Moraes CL, Mello Jorge $\mathrm{MH}$, Silva CM, Minayo MC. Violence and injuries in Brazil: the effect, progress made, and challenges ahead. Lancet 2011; 377(9781):1962-1975.

4. Veras R. Living a full life without violence at maturity: the contemporary search. Cien Saude Colet 2010; 15(6):2671-2673.

5. Castro VC, Rissardo LK, Carreira L. Violence against the Brazilian elderlies: an analysis of hospitalizations. Rev Bras Enferm 2018; 71(2):830-838.

6. Espíndola CR, Blay SL. Prevalence of elder abuse: a systematic review. Rev Saude Publica 2007; 41(2):301306.

7. Yon Y, Miktron CR, Gassoumis ZD, Wilber KH. Elder abuse prevalence in Community settings: a systematic review and meta-analysis. Lancet Glob Health 2017; 5(2):147-156.

8. Organización Mundial de la Salud (OMS). Declaración de Toronto para la prevención global del maltrato de lãs personas mayores. Ginébra: OMS; 2003.

9. Faustino AM, Gandolfi L, Moura LBA. Functional capability and violence situations against the elderly. Acta Pauli Enferm 2014; 27(5):392-398.

10. Oliveira AAV, Trigueiro DRSG, Fernandes MGM, Silva AO. Elderly maltreatment: integrative review of the literature. Rev Bras Enferm 2013; 66(1):128-133.

11. Patrick DL, Erickson P. Health Status and health policy - quality of life in health care evaluation and resource allocation. New York: Oxford University Press; 1993.

12. Wilson IB, Cleary PD. Linking clinical variables with health-related quality of life. A conceptual model of patient outcomes. JAMA 1995; 273(1):59-65.

13. Sorensen J, Kruse M, Gudex C, Helweg-Larsen K, Bronnum-Hansen H. Physical violence and health-related quality of life: Danish cross-sectional analyses. Health Qual Life Outcomes 2012; 10(113):110-119.

14. Costa D, Hatzidimitriadou E, Ioannidi-Kapolou E, Lindert J, Soares J, Sundin O, Toth O, Barros H. Intimate partner violence and health-related quality of life in European men and women: findings from the DOVE study. Qual Life Res 2015; 24(2):463-471.

15. Gharacheh M, Azadi S, Mohammadi N, Montazeri S, Khalajinia Z. Domestic Violence During Pregnancy and Women's Health-Related Quality of Life. Glob J Health Sci 2015; 8(2):27-34.

16. Ribeiro KT. Fatores associados à qualidade de vida relacionada à saúde de idosos residentes no Município de São Paulo - Estudo SABE: Saúde, Bem-Estar e Envelhecimento [tese]. São Paulo: Faculdade de Saúde Pública; 2011.

17. Camelo LV, Giatti L, Barreto, M. Health related quality of life among elderly living in region of high vulnerability for health in Belo Horizonte, Minas Gerais, Brazil. Rev Bras Epidemiol 2016; 19(2):280-293.

18. Palloni A, Pelaez M. SABE: Survey on Health and Well-being of Elders - preliminary report [internet] 2002 Nov [cited 2018 Fev 5]: [about 102 p.]. Available from: www.ssc.wisc.edu/sabe/docs/informeFinal $\% 20$ Ingles\%20noviembre\%202004
19. Lebrão ML, Laurenti R. Health, well-being and aging: the SABE study in São Paulo, Brazil. Rev Bras Epidemiol 2005; 8(2):127-141.

20. Tamanini JTN, Sartori MGR, Santos JLF, Kerrebroeck PEVA, Pallone LV, Girão MJBC, Duarte YAO. A populational based survey on the prevalence, incidence, and risk factors of urinary incontinence in older adults - results from the "SABE STUDY. Neurourol Urodyn 2018; 37(18):466-477.

21. Brasil. Ministério da Saúde (MS). Secretaria Executiva. DATASUS, 2017. [acessado 2017 Jun 18]. Disponível em: http://www.datasus.gov.br.

22. Ware JE, Kosinski MA, Keller SD. A 12-Item ShortForm Health Survey - construction of scales and preliminary tests of reliability and validity. Med Care 1996; 34(3):220-233.

23. Guedes DT, Curcio CL, Liano BA, Zunzunegui MV, Guerra R. The gender gap in domestic violence in older adults in Latin America: the IMIAS Study. Rev Panam Salud Publica 2015; 37(4-5):293-300.

24. Andrade TL, Camelier AA, Rosa FW, Santos MP, Jezler S, Silva JLP. Applicability of the 12-Item Short-Form Health Survey in patients with progressive systemic sclerosis. J Bras Pneumol 2007; 33(4):414-422.

25. Silveira MF, Almeida JC, Freire RS, Haikal DS, Martins AEBL. Psychometric properties of the quality of life assessment instrument: 12-item health survey (SF12). Cien Saude Colet 2013; 18(7):1923-1931.

26. Ware JE, Sherbourne CD. The MOS 36-item ShortForm Health Survey (SF-36). Med Care 1992 30(6):473-483.

27. Ciconelli RM, Ferraz MB, Santos W, Meinão I, Quaresma MR. Tradução para a língua portuguesa e validação do questionário genérico de qualidade de vida SF-36 (Brasil SF-36). Rev Bras Reumatol 1999; 39(3):143-150.

28. Folstein MF, Folstein SE, Mchugh PR. Mini-Mental State - A Practical Method for Grading the Cognitive State of Patients for the Clinician. J Psychiatr Res 1975; 12(3):189-198.

29. Cerqueira ATAR. Deterioração cognitiva e depressão. In: Lebrão ML, Duarte YAO, organizadores. SABE Saúde, Bem-Estar e Envelhecimento - O Projeto SABE no Município de São Paulo: uma abordagem inicial. Brasília: Organização Pan-Americana de Saúde; 2003. p. 141-165.

30. Bertolucci PHF, Brucki SMD, Campacci SR, Juliano Y. O Mini-Exame do Estado Mental em uma população geral: impacto da escolaridade. Arq Neuropsiquiatr 1994; 52(1):1-7.

31. Almeida OP, Almeida SA. Confiabilidade da versão brasileira da escala de depressão em geriatria (GDS) versão reduzida. Arq Neuropsiquiatr 1999; 57(2):421426.

32. Duarte YAO. Família: rede de suporte ou fator estressor. A ótica de idosos e cuidadores familiares [tese]. São Paulo: Universidade de São Paulo; 2001.

33. Katz S, Ford AB, Moskowitz RW, Jackson BA, Jaffe MW. Studies of illness in the aged. The index of ADL a standardized measure of biological and psychosocial function. JAMA 1963; 21(185):914-919.

34. Lawton MP, Brody EM. Assessment of older people Self-maintaining and instrumental activities of daily living. Gerontologist 1969; 9(3):179-186. 
35. Duque AM, Leal MCC, Marques APO, Eskinazi FMV, Duque AM. Violence against the elderly in the home environment: prevalence and associated factors (Recife, State of Pernambuco). Cien Saude Colet 2012; 17(8):2199-2208.

36. Bolsoni CC, Coelho EBS, Giehl MWC, d'Orsi E. Prevalence of violence against the elderly and associated factors - a population based study in Florianópolis, Santa Catarina. Rev Bras Geriatr Gerontol 2016; 19(4):671-682.

37. Schneider IJC, Confortin SC, Bernardo CO, Bolsoni CC, Antes DL, Pereira KG, Ono LM, Marques LP, Borges LJ, Gieh MWC, Krug RR, Goes VF, Boing AC, Boing AF, d'Orsi E. Estudo de coorte EpiFloripa Idoso: métodos, aspectos operacionais e estratégias de seguimento. Rev Saude Publica [periódico na Internet]. 2017 Nov [acessado $2018 \mathrm{Fev} 16$ ]; 51(104): [cerca de 10 p.]. Disponível em: http://www.scielo.br/pdf/rsp/ v51/pt_0034-8910-rsp-S1518-87872017051006776. pdf

38. Brasil. Ministério da Saúde (MS). Envelhecimento e saúde da pessoa idosa - Cadernos de Atenção Básica. Brasília: MS; 2006.

39. Hair JF, Black WC, Babin BJ, Anderson RE, Tatham RL. Análise multivariada de dados. $6^{a}$ ed. Porto Alegre: Bookman; 2009.

40. Blay SL, Laks J, Marinho V, Figueira I, Maia D, Coutinho ESF, Quintana IM, Mello MF, Bressan RA, Mari JJ, Andreoli SB. Prevalence and Correlates of Elder Abuse in São Paulo and Rio de Janeiro. J Am Geriatr Soc 2017; 65(12):2634-2628.

41. Moraes CL, Apratto Júnior PC, Reichenheim ME. Breaking silence and its barriers: a household survey on domestic violence against the elderly within the scope of a Family Health Program in Niterói, Rio de Janeiro State, Brazil. Cad Saude Publica 2008; 24(10):2289-2300.

42. Santos SM, De Marchi RJ, Martins AB, Hugo FN, Padilha DM, Hilgert JB. The prevalence of elder abuse in the Porto Alegre metropolitan area. Braz Oral Res 2013; 7(3):197-202.

43. Paiva MM, Tavares DMS. Physical and psychological violence against the elderly: prevalence and associated factors. Rev Bras Enferm 2015; 68(6):1035-1041.

44. Melo VL, Cunha JOC, Falbo Neto GH. Maus-tratos contra idosos no município de Camaragibe, Pernambuco. Rev Bras Saude Mater Infant 2006; 6(1):43-48.

45. Sooryanarayana R, Choo WY, Hairi NN. A Review on the prevalence and measurement of elder abuse in the community. Trauma Violence Abuse 2013; 14(4):316325.

46. Gomez Ricardez LA, Abrego GR, Llamas EK. Prevalencia y factores asociados a violencia familiar en adultos mayores de Ocozocoautla (Chiapas, México). Rev Esp Geriatr Gerontol 2007; 42(1):27-34.
47. Abdel Rahman TT, El Gaafary MM. Elder mistreatment in a rural area in Egypt. Geriatr Gerontol Int 2012; 12(3):532-537.

48. Yadav UN, Paudel G. Prevalence and associated factors of elder mistreatment: A cross sectional study from urban Nepal. Age Ageing 2016; 45(5):609-613.

49. Neri AL. Idosos no Brasil - vivências, desafios e expectativas na terceira idade. São Paulo: Editora Fundação Perseu Abramo, Edições SESC-SP; 2007.

50. Brasil. Ministério da Saúde (MS). Impacto da violência na saúde dos brasileiros. Brasília: MS; 2005.

51. Park HJ. Living with 'Hwa-byung': the psycho-social impact of elder mistreatment on the health and well-being of folder people. Aging Ment Health 2014; 18(1):125-128.

52. Naughton C, Drennan J, Lyons I, Lafferty A, Treacy M, Phelan A, O'Loughlin A, Delaney L. Elder abuse and neglect in Ireland: results from a national prevalence survey. Age Ageing 2012; 41(1):98-103.

53. De Belvis AG, Avolio M, Sicuro L, Rosano A, Latini E, Damiani G, Ricciardi W. Social relationships and HRQL: A cross-sectional survey among older Italian Adults. BMC Public Health 2008; 8(348):1-16.

54. Wranker LS, Rennemark M, Berglund J, Elmstahl S. Relationship between pain and quality of life-Findings from the Swedish National study on Aging and Care. Scand J Pain 2014; 5(4):270-275.

55. Cunha LL, Mayrink WC. Influência da dor crônica na qualidade de vida em idosos. Rev Dor 2011; 12(2):120-124.

56. Sallum AMC, Garcia DM, Sanches M. Dor aguda e crônica: revisão narrativa da literatura. Acta Paul Enferm 2012; 25(1):150-154.

57. Bretanha AF, Facchini LA, Nunes BP, Munhoz TN, Tomasi E, Thumé E. Depressive symptoms in elderly living in áreas covered by Primary Health Care Units in urban area of Bagé, RS. Rev Bras Epidemiol 2015; 18(1):1-12.

Artigo apresentado em 31/03/2018

Aprovado em 28/07/2018

Versão final apresentada em 30/07/2018 